\title{
AVALIAÇÃO DA COLMATAÇÃO APÓS 15 ANOS DE OPERAÇÃO DE UM SISTEMA HÍBRIDO DE WETLAND
} CONSTRUÍDO

\author{
Rafael Favretto $^{1}$, Cristiane Lisboa Giroletti ${ }^{2}$, Maria Elisa Magri ${ }^{2}$, Carla Suntti $^{3}$ \\ 1Departamento de Engenharia Sanitária e Ambiental, Universidade do Oeste de Santa Catarina, 89560-000, Videira, Brasil. \\ ${ }^{2}$ Departamento de Engenharia Sanitária e Ambiental da Universidade Federal de Santa Catarina, Florianópolis, 88040-900, \\ Florianópolis, Brasil. \\ ${ }^{3}$ Departamento de Engenharia Química e Engenharia Sanitária e Ambiental, Universidade do Oeste de Santa Catarina, 89560-000, \\ Videira, Brasil \\ *E-mail: rafaelfjba@hotmail.com
}

\section{RESUMO}

O objetivo deste trabalho foi avaliar as condições iniciais de colmatação e o funcionamento de um sistema híbrido de wetland construído (vertical + horizontal), já em operação há 15 anos recebendo esgoto proveniente do Centro de Treinamento de Videira - SC (CETREVI). O período de monitoramento foi de agosto até novembro de 2016. Durante esse período, foram realizadas atividades de limpeza do sistema e de reintrodução das macrófitas, implementando-se uma nova rotina de alimentação do wetland vertical. No efluente,

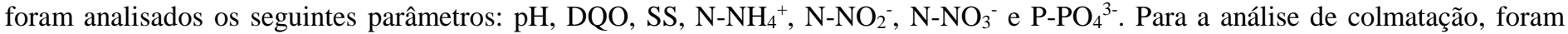
coletadas amostras do leito do wetland horizontal, as quais foram encaminhadas ao laboratório para secagem, peneiramento e posterior determinação dos sólidos finos e grosseiros. Embora o sistema apresentasse indícios de colmatação, as características físico-químicas do efluente permaneceram dentro dos padrões estabelecidos pela legislação vigente, com exceção apenas dos parâmetros $\mathrm{N}-\mathrm{NO}_{2}{ }^{-}$e N-NO${ }_{3}^{-}$. Quanto aos sólidos de colmatação, verificou-se que o primeiro terço do leito apresentou grandes quantidades de sólidos orgânicos, uma ocorrência que pode ser explicada pelo fato de essa parte do leito ser a entrada do efluente no sistema. Já no segundo e terceiro terços do leito essa quantidade diminuiu, mas não de forma acentuada.

Palavras-chave: Tratamento de esgoto. Wetland construído. Sistema híbrido. Colmatação.

\section{Introdução}

Informações do relatório Progress on Drinking Water, Sanitation and Hygiene apontam que, no ano de 2015, no Brasil, $91 \%$ das residências de áreas urbanas possuíam instalações sanitárias (redes coletoras de esgoto, ou tanques sépticos). Em residências localizadas nas áreas rurais, no entanto, esse percentual era de apenas $58 \%$ [1]. Diante dos dados apresentados, fica nítida a proporção do descaso do governo brasileiro com as condições de saneamento básico da população rural.

Desenvolver e expandir o uso de outras formas de destinação adequada do esgoto doméstico em áreas rurais são de fundamental importância para a mudança do quadro atual do saneamento brasileiro. Uma das formas de sanar essa lacuna é a utilização de sistemas descentralizados para tratamento de águas residuárias. Entre os sistemas existentes, encontram-se os wetlands construídos, os quais, no Brasil, têm sido utilizados com sucesso para o tratamento de esgoto em regiões com baixa densidade populacional [2].

Os wetlands construídos são utilizados mundialmente para o tratamento de águas residuárias, pois possibilitam a remoção de matéria orgânica, nutrientes e microrganismos. Esse sistema permite ainda a obtenção de efluentes com parâmetros de qualidade superior, quando comparados a tratamentos primários. Ademais, devido a seu baixo custo de implantação e manutenção, e sua operação simplificada [3], o sistema apresenta alta viabilidade econômica e ambiental para as áreas rurais.

Por serem considerados "filtros", é evidente que uma das principais funções de tais sistemas é reter a fração sólida presente no efluente. Essa retenção de partículas sólidas e de matéria orgânica, somada a longos períodos sem manutenção e limpeza, podem causar condições de "entupimento", processo também conhecido como colmatação, o qual, por fim, confere ao efluente uma qualidade inferior, quando comparado a sistemas não colmatados. Essas condições reduzem a capacidade de filtração do 
wetland que, por conseguinte, propicia um declínio do processo de purificação dos contaminantes [4].

$\mathrm{O}$ processo de colmatação em wetlands ocorre devido à ação dos cinco seguintes fatores: a) deposição de sólidos suspensos inertes (mineral) na região de entrada do leito; b) acumulação de material orgânico refratário (resistente à degradação microbiana) na zona de entrada do leito; c) deposição de precipitados químicos no leito do wetland; d) carregamento de matéria orgânica (suspensa e dissolvida) que estimula o crescimento microbiano do biofilme do leito; e e) desenvolvimento de redes de raízes de plantas que ocupam o volume dos poros dentro do leito [5].

No Centro de Treinamento de Videira (CETREVI pertencente à EPAGRI - Empresa de Pesquisa Agropecuária e Extensão Rural do Estado de Santa Catarina), em 2001, foi implantada a estação de tratamento de efluentes (ETE), constituída por um tanque séptico e um wetland horizontal de fluxo subsuperficial. Em 2010 a ETE foi aprimorada com a instalação de um wetland vertical, a fim de melhorar a característica do efluente com relação à matéria nitrogenada. No entanto, por falta de continuidade da operação e de monitoramento da ETE, a bomba que alimentava o wetland vertical não foi acionada, fazendo com que o efluente seguisse diretamente ao wetland horizontal em vez de ser direcionado ao wetland vertical. Essa alteração na sequência do tratamento resultou em indícios de colmatação (obstrução da passagem do efluente pelo meio filtrante) no wetland horizontal e na morte da grande maioria das macrófitas, povoando o wetland vertical com espécies daninhas.

Diante do exposto, este estudo tem como objetivo avaliar a colmatação do wetland horizontal, caracterizando seu meio suporte, bem como, física e quimicamente, analisar o efluente gerado em todas as etapas do tratamento, visando uma proposta de medidas para aumentar e eficiência da ETE.

\section{Metodologia}

\subsection{Descrição do local de estudo}

Esta pesquisa foi desenvolvida e realizada no CETREVI da EPAGRI, localizada na cidade de Videira - SC (latitude $27^{\circ} 00^{\prime} 30^{\prime \prime}$, longitude $51^{\circ} 09^{\prime} 06^{\prime \prime}$ e altitude de $750 \mathrm{~m}$ acima do nível do mar).

A ETE, localizada nas proximidades do CETREVI, recebe o efluente do Centro, constituído pela somatória das águas negras provenientes dos banheiros, cozinha e refeitório, e das águas cinzas da lavanderia, lavatórios e chuveiros.

$\mathrm{Na}$ Figura 1, pode-se visualizar a disposição física da ETE, a qual é composta por um tanque séptico (TS), um reservatório de equalização (EQ), um wetland construído de fluxo vertical (WV), plantado com as espécies Taboa (Typha spp.) e Junco (Juncos spp.), e um wetland construído de fluxo horizontal (WH). O efluente final é infiltrado no solo.

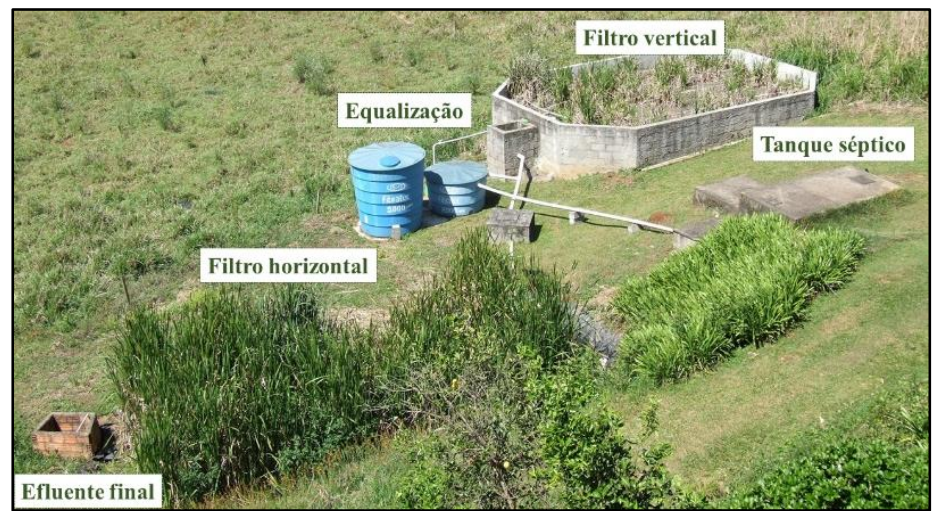

Figura 1 - Estação de tratamento de efluentes do CETREVI.

As características do sistema são apresentadas na Tabela 1.

Tabela 1 - Principais dimensões do sistema de tratamento de esgoto.

\begin{tabular}{lccc}
\hline \multicolumn{4}{l}{ Tabela 1 - Principais dimensões do sistema de tratamento de esgoto. } \\
Dimensões & $\begin{array}{c}\text { Tanque } \\
\text { séptico }\end{array}$ & $\begin{array}{c}\text { Filtro } \\
\text { vertical }\end{array}$ & $\begin{array}{c}\text { Filtro } \\
\text { horizontal }\end{array}$ \\
\hline Comprimento (m) & 3,30 & 9,60 & 10,00 \\
Largura (m) & 1,75 & 6,60 & 5,00 \\
Altura (m) & 2,25 & 1,05 & 0,70 \\
Altura do leito (m) & - & 0,60 & 0,70 \\
Volume (m $\left.{ }^{3}\right)$ & 13,00 & - & - \\
Área superficial $\left(\mathrm{m}^{2}\right)$ & - & 63,00 & 50,00 \\
\hline
\end{tabular}

\subsection{Amostragem e análise do efluente}

As análises do efluente foram realizadas no período entre agosto e novembro de 2016. O processo de monitoramento do efluente é indispensável nos estudos de colmatação dos filtros plantados, uma vez que o escoamento superficial causado pela colmatação altera a qualidade final do efluente. Para isso, foram avaliados os seguintes parâmetros: DQO (Demanda química de oxigênio) pelo método de digestão em refluxo fechado, com leitura colorimétrica; nitrogênio amoniacal $\left(\mathrm{N}^{-\mathrm{NH}_{4}}{ }^{+}\right)$; nitrogênio nitrito $\left(\mathrm{N}-\mathrm{NO}_{2}{ }^{-}\right)$; nitrogênio nitrato $\left(\mathrm{N}-\mathrm{NO}_{3}{ }^{-}\right)$; ortofosfato $\left(\mathrm{P}-\mathrm{PO}_{4}{ }^{3-}\right)$ pelo método da determinação colorimétrica; potencial hidrogeniônico $(\mathrm{pH})$ pelo método potenciométrico; e sólidos suspensos (SS) pelo método de filtração e secagem a uma temperatura de $105^{\circ} \mathrm{C}$. Todas as coletas e análises realizadas com o efluente seguiram as metodologias descritas em Standard Methods for Examination of Water and Wastewater [6] .

\subsection{Estimativa da vazão}

Para estimar a vazão do sistema de tratamento de esgoto foram solicitados relatórios mensais das visitações ao centro (com e sem pernoite) e o número total de funcionários. Os dados apurados foram então estudados utilizando coeficientes de consumo per capita de água, conforme descrito na literatura. Para um hotel sem per noite, adota-se um coeficiente de consumo de 
água entre 30 e $49 \mathrm{~L} \mathrm{dia}^{-1}$ hóspede ${ }^{-1}$. E para um hotel com pernoite, considera-se um consumo de 151 a $227 \mathrm{~L} \mathrm{dia}^{-1}$ hóspede $^{-1}$ [7]. A estimativa da vazão do centro foi calculada pela Equação (1), conforme dados da literatura [8].

$$
Q=\frac{C x p x q}{86400}
$$

Equação (1)

Onde:

$\mathrm{Q}=$ Vazão de esgoto $\left(\mathrm{L} \mathrm{s}^{-1}\right)$

$\mathrm{C}=$ Contribuição de esgoto

$\mathrm{p}=$ População (hab)

$\mathrm{q}=$ Consumo efetivo de água per capita $\left(\mathrm{L} \mathrm{hab}^{-1} \mathrm{dia}^{-1}\right)$

A contribuição de esgoto considerada para os visitantes foi a média dos valores de referência calculada em $264,5 \mathrm{~L} \mathrm{dia}^{-1}$ hóspede ${ }^{-1}$, com pernoite, e em 54,5 $\mathrm{L} \mathrm{dia}^{-1}$ hóspede ${ }^{-1}$, sem pernoite [7]. Considerando-se as atividades dos visitantes, o coeficiente de retorno adotado foi de 0,80 [9].

\subsection{Caracterização do material filtrante}

A caracterização do grau de colmatação no sistema foi realizada através de coletas no leito do wetland [10-11]. As amostras do leito foram coletadas a uma profundidade entre $0 \mathrm{e}$ $20 \mathrm{~cm}$ e entre 20 e $35 \mathrm{~cm}$, conforme a Figura 2.

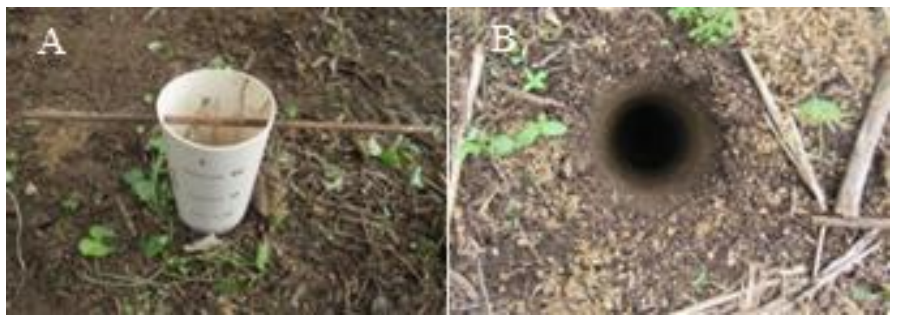

Figura 2 - Coletor cravado no leito (a) e leito após a retirada do coletor (b).

A fim de avaliar as alterações de acúmulo de sólidos no leito, foram feitas duas coletas; uma no período inicial de monitoramento e outra no período final. Em cada uma das coletas foram amostrados seis pontos alocados no leito.

As amostras foram encaminhadas para análise em laboratório, passando por um período de secagem sob temperatura ambiente, seguido por um peneiramento [12]. As peneiras utilizadas apresentavam malhas de 9,50; 4,75; 2,36; 2,00; 1,00 e $0,212 \mathrm{~mm}$. Após o peneiramento, os materiais retidos nas peneiras (com malhas de 9,50;4,75;2,36;2,00 e 1,00 mm) foram lavados com aproximadamente 1,5 litro de água destilada, a fim de remover os sólidos intersticiais retidos no material. O líquido resultante desta lavagem foi transferido para béqueres de dois litros e acondicionado sobre chapas de aquecimento a uma temperatura de $65^{\circ} \mathrm{C}$, até que o volume dos béqueres resultasse num resíduo com aspecto de "lodo" e/ou "lama". Depois dessa redução, as amostras foram colocadas numa estufa a uma temperatura de $65^{\circ} \mathrm{C}$, por 24 horas, para concluir a secagem. $\mathrm{O}$ material resultante dessa secagem foi destorroado e novamente peneirado usando uma peneira com malha de 0,212 mm. Após o peneiramento, o material foi pesado e juntou-se às amostras iniciais da mesma granulometria.

As amostras de material do leito do filtro foram coletadas com o objetivo de quantificar e caracterizar os sólidos intersticiais, os quais foram divididos em sólidos grosseiros (diâmetro das partículas entre 0,212 e 1,0 mm) e sólidos finos (diâmetro das partículas menor que $0,212 \mathrm{~mm}$ ). As análises realizadas com os sólidos grosseiros e sólidos finos foram: sólidos totais (ST), pelo método gravimétrico, com secagem a uma temperatura de $105^{\circ} \mathrm{C}$; sólidos voláteis totais (SVT); e sólidos fixos totais (SFT), obtidos após a calcinação do material em mufla, a uma temperatura de $550{ }^{\circ} \mathrm{C}$, por duas horas [6].

\section{Resultados e discussões}

\subsection{Estimativa de vazão}

A contagem do número de pessoas que frequentam o Centro foi considerada de extrema importância para este trabalho, uma vez que esse dado compõe a estimativa da vazão gerada pelo centro. Utilizando-se a Equação (1), obteve-se uma estimativa de vazão igual a $9,5 \mathrm{~m}^{3} \mathrm{dia}^{-1}$. No ano de 2013, a vazão obtida foi de $2,9 \mathrm{~m}^{3} \mathrm{dia}^{-1}$ [13]. Essa variação na vazão é consequência da grande oscilação no número de pessoas que frequentam o Centro de Treinamento e das atividades que lá são desenvolvidas.

\subsection{Desempenho do sistema de tratamento}

A caracterização do efluente é apresentada na Tabela 2, sendo que os valores médios das concentrações dos parâmetros físico-químicos foram obtidos nos seguintes pontos de amostragem do sistema de tratamento: efluente bruto, saída do tanque séptico, saída do wetland vertical e saída do wetland horizontal.

Conforme se pode observar na Tabela 2, a remoção global do sistema para os parâmetros DQO, $\mathrm{SS}, \mathrm{N}-\mathrm{NH}_{4}{ }^{+}$e $\mathrm{P}-\mathrm{PO}_{4}{ }^{3-}$ foi de $90 \%, 100 \%, 71 \%$ e $84 \%$, respectivamente. Em 2013, durante um monitoramento realizado no mesmo sistema e com os mesmos parâmetros, o percentual de remoções foi de $89 \%, 79 \%, 92 \%$ e $89 \%$, respectivamente [13]. Como se pode notar, a remoção de SS foi mais eficiente neste trabalho, enquanto as remoções de $\mathrm{N}-\mathrm{NH}_{4}{ }^{+}$ e $\mathrm{P}_{-} \mathrm{PO}_{4}{ }^{3-}$ apresentaram uma menor eficiência do que no monitoramento de 2013. 
Tabela 2. Características físico-químicas do efluente em todas as etapas de tratamento.

\begin{tabular}{|c|c|c|c|c|}
\hline $\begin{array}{c}\text { Etapa do } \\
\text { tratamento }\end{array}$ & $\begin{array}{l}\text { Efluente } \\
\text { bruto } \\
\text { Média } \\
\pm \text { DP }\end{array}$ & $\begin{array}{l}\text { Saída do } \\
\text { tanque } \\
\text { séptico } \\
\text { Média } \\
\pm \text { DP }\end{array}$ & $\begin{array}{c}\text { Saída do } \\
\text { wetland } \\
\text { vertical } \\
\text { Média } \\
\pm \text { DP }\end{array}$ & $\begin{array}{c}\text { Saída do } \\
\text { wetland } \\
\text { horizonta } \\
\text { Média } \\
\pm \text { DP }\end{array}$ \\
\hline \multicolumn{5}{|l|}{$\begin{array}{l}\text { Parâmetros } \\
\left(\mathrm{mg} \mathrm{L}^{-1}\right) \\
(\mathrm{n}=4)\end{array}$} \\
\hline $\mathrm{pH}$ & 6,6 & 6,8 & 6,7 & 6,3 \\
\hline DQO & $\begin{array}{c}649,0 \pm \\
271,8\end{array}$ & $\begin{array}{c}518,5 \pm \\
199,3\end{array}$ & $\begin{array}{c}121,2 \pm \\
38,5\end{array}$ & $65,6 \pm 34$ \\
\hline SS & $\begin{array}{c}225,9 \pm \\
144,0\end{array}$ & $\begin{array}{c}133,2 \pm \\
103,0\end{array}$ & $3,1 \pm 2,4$ & $0,0 \pm 0,0$ \\
\hline $\mathrm{N}-\mathrm{NH}_{4}{ }^{+}$ & $19,1 \pm 3,4$ & $14,5 \pm 6,7$ & $10,4 \pm 4,4$ & $5,5 \pm 1,2$ \\
\hline $\mathrm{N}-\mathrm{NO}_{3}^{-}$ & $0,3 \pm 0,1$ & ND & $\mathrm{ND}$ & $19,57 \pm 5$ \\
\hline $\mathrm{N}-\mathrm{NO}_{2}^{-}$ & $0,09 \pm 0,1$ & $0,05 \pm 0,1$ & $0,01 \pm 0,1$ & $0,03 \pm 0,1$ \\
\hline $\mathrm{P}-\mathrm{PO}_{4}{ }^{3-}$ & $33,9 \pm 5,9$ & $23,0 \pm 11,6$ & $14,1 \pm 9,9$ & $5,4 \pm 5,6$ \\
\hline \multicolumn{5}{|c|}{ Legenda: n: número de amostras; ND: não detectável - Abaixo do limite de detecção. } \\
\hline \multicolumn{5}{|c|}{$\begin{array}{l}\text { sólidos suspensos, pode-se constatar que o wetland de fluxo } \\
\text { vertical está realizando sua função quanto à remoção de DQO e } \\
\text { SS, atingindo uma remoção de } 77 \% \text { e } 98 \% \text {, respectivamente. No } \\
\text { entanto, verifica-se uma ligeira redução no processo de conversão } \\
\text { de amônia e ortofosfato no wetland vertical. Tal redução está } \\
\text { associada a vários fatores (como por exemplo, o tempo de } \\
\text { detenção e uma concentração de matéria orgânica elevada), os } \\
\text { quais fazem com que os microrganismos responsáveis por esses } \\
\text { processos de conversão reduzam suas atividades. }\end{array}$} \\
\hline
\end{tabular}

\subsection{Avaliação do grau de colmatação do wetland horizontal de fluxo subsuperficial}

Em wetlands de fluxo subsuperficial a acumulação e decomposição de partículas de matéria orgânica nos espaços vazios do leito é a principal forma de contribuição para a colmatação [14]. Neste estudo, a avaliação da colmatação do wetland foi feita através do método direto, o que permite apresentar os teores de sólidos totais, voláteis e fixos nas amostras dos sólidos finos e grosseiros, responsáveis pela colmatação.

Também foi possível perceber que as condições de colmatação encontradas foram propiciadas pelos sólidos de origem orgânica (gerados pela decomposição das plantas do sistema) e pela deposição de SS do próprio efluente.

Na Figura 3, é possível perceber que, após a retirada do coletor, houve uma estabilidade do material filtrante; estabilidade essa que evidencia a presença de grandes quantidades de sólidos de colmatação. Esse comportamento de "cimentação" é ocasionado pela coesão do substrato [10-15]. Ademais, vale ressaltar que a colmatação do meio poroso está associada a fatores tais como a quantidade de sólidos intersticiais, as características desses sólidos e a presença ou não de plantas, assim como à interação complexa que ocorre entre esses fatores [11]. Os resultados referentes aos sólidos de origem orgânica são representados pela fração de SV (sólidos voláteis) e estão apresentados nas Figuras 3 e 4.

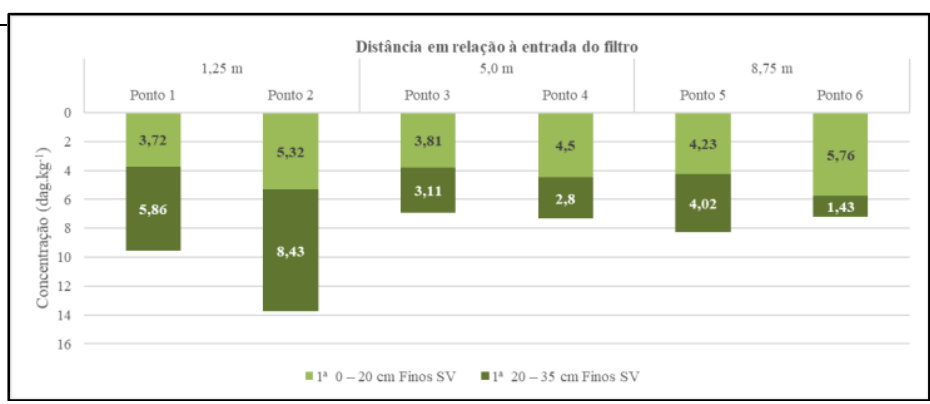

Figura 3 - Sólidos voláteis (SV) finos na primeira amostragem.

Legenda: SV Finos: sólidos voláteis finos.

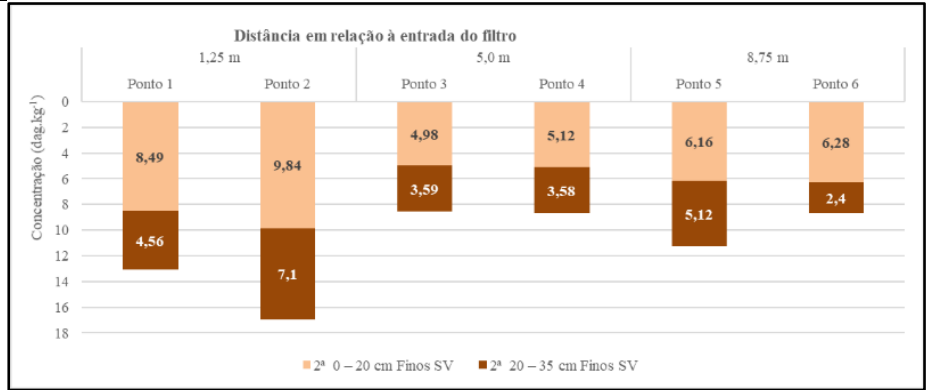

Figura 4 - Sólidos voláteis (SV) finos na segunda amostragem

Legenda: SV Finos: sólidos voláteis finos.

Com uma análise mais aprofundada desses dados, podese observar que há uma maior quantidade de matéria orgânica no primeiro terço do leito. Observa-se também que, no segundo e terceiro terços do leito, essa quantidade diminui, porém, não de forma acentuada. Tais condições são decorrentes da grande quantidade de plantas que estavam em decomposição e, com isso, contribuíram para a maior quantidade de matéria orgânica do sistema [16].

Conforme os resultados apresentados nas Figuras 3 e 4, pode-se verificar ainda que houve uma ligeira diferença entre os valores de material fino encontrados. Esses sólidos voláteis finos são caracterizados como sólidos de colmatação, uma vez que apresentam um diâmetro de partícula menor que os grosseiros e, dessa forma, acabam causando o entupimento dos poros do leito filtrante [17].

Comparações entre teores de sólidos de colmatação em wetlands, plantados e não plantados, identificam que os sólidos voláteis finos são de 2,6 a 3,1 vezes maiores nos wetlands plantados do que nos sistemas não plantados. Os depósitos de material orgânico gerados pelas plantas contribuem de forma significativa para a colmatação do filtro [15].

De maneira geral, percebe-se que os dados tiveram variação por toda a extensão do leito e também nas duas 
profundidades de coleta. Isso fica mais evidente quando da avaliação dos resultados do primeiro terço do leito $(1,25 \mathrm{~m})$, aonde é possível visualizar as grandes quantidades de sólidos voláteis atuando na obstrução dos poros.

Como solução para a problemática de colmatação do sistema monitorado neste estudo, recomenda-se que seja feita a troca da areia do meio filtrante. Para tal, pode ser feita a substituição de todo o leito, utilizando-se uma areia com granulometria maior e mais uniforme, ou até mesmo a substituição parcial, removendo apenas a camada do primeiro terço do filtro que, conforme a segunda amostragem apresentada na Figura 3, é a camada com maior quantidade de sólidos voláteis finos.

\section{Conclusões}

Com os resultados obtidos neste estudo pode-se concluir que, mesmo o sistema apresentando indícios de colmatação, as características físico-químicas do efluente estiveram dentro dos padrões estabelecidos pela legislação vigente, com exceção apenas dos parâmetros $\mathrm{N}_{-} \mathrm{NO}_{2}^{-}$e $\mathrm{N}^{-} \mathrm{NO}_{3}^{-}$. Acredita-se que o processo tardio da nitrificação tenha influenciado os resultados. Em relação a colmatação, a utilização do método direto de identificação é eficiente para a quantificação de sólidos voláteis, porém, outras metodologias podem ser adotadas para efeitos de comparação de resultados. A colmatação se manteve a mesma com a recolocação de plantas no sistema dentro do período de avaliação desta pesquisa. Para que se possa elucidar o papel do replantio, sugere-se que seja feito um monitoramento dessa situação por um maior período de tempo.

\section{Agradecimentos}

Nossos sinceros agradecimentos aos responsáveis do CETREVI, pelo apoio no desenvolvimento do trabalho, e ao governo do Estado de Santa Catarina pela concessão da bolsa, por meio do Artigo 170.

\section{ASSESSMENT OF INITIAL CLOGGING IN A CONSTRUCTED WETLAND HYBRID SYSTEM AFTER 15 YEARS OF OPERATION}

ABSTRACT: The purpose of this research paper is to assess initial clogging conditions and performance of a constructed wetland hybrid system in operation for the past 15 years, receiving sewage from the Training Center in Videira - State of Santa Catarina (CETREVI, in the Portuguese abbreviation). The monitoring process took place between August and November of 2016. In that period, the system was cleaned, macrophytes were reintroduced, and new procedures were implemented for the flow of wastewater into the vertical wetland. The wastewater was analyzed for the following parameters: $\mathrm{pH}, \mathrm{COD}, \mathrm{SS}, \mathrm{N}-\mathrm{NH}_{4}{ }^{+}, \mathrm{N}-$ $\mathrm{NO}_{2}^{-}, \mathrm{N}-\mathrm{NO}_{3}{ }^{-}$, and $\mathrm{P}_{-} \mathrm{PO}_{4}{ }^{3-}$. In order to analyze the clogging conditions, samples were collected from the bed of the horizontal wetland, and sent to a laboratory to be dried and sifted, so its composition of fine and coarse solids could be determined. In spite of the fact that the system indeed showed signs of clogging, wastewater physical-chemical characteristics were still within the standards established by current Brazilian legislation, the only exceptions being that for parameters $\mathrm{N}_{-} \mathrm{NO}_{2}{ }^{-}$and $\mathrm{N}-\mathrm{NO}_{3}{ }^{-}$. When considering the solids that can cause clogging, it was determined that the first third of the wetland bed contained large quantities of organic solids, an occurrence that can be explained by the fact that that layer of the bed is the wastewater's point of entrance into the system. In the second and third layers of the wetland bed, the quantities of organic solids decreased, but not significantly.

Keywords: Sewage treatment. Constructed wetlands. Hybrid system. Clogging.

\section{Referências}

[1] UNICEF, World Health Organization (WHO). Progress on drinking water and sanitation: 2017 update. Geneva: Unicef, WHO: 2017

[2] MACHADO, A.I.; BERETTA, R.; FRAGOSO, R.; DUARTE, E. Overview of the state of the art of constructed wetlands for decentralized wastewater management in Brazil, Journal of Environmental Management. Vol 187, p. 560$570,2016$.

[3] SEZERINO, P.H. Potencialidade dos filtros plantados com macrófitas (constructed wetlands) no pós-tratamento de lagoas de estabilização sob condições de clima subtropical. 2006. 171 f. Tese (Programa de Pós Graduação em Engenharia Ambiental- Doutorado) Universidade Federal de Santa Catarina, Florianópolis. 2006.

[4] FU, G.; ZHANG, J.; CHEN, W.; CHEN, Z. Medium clogging and the dynamics of organic matter accumulation in constructed wetlands. Ecological Engineering. Vol. 60, p. 393-398, 2013.

[5] KADLEC, R.H.; WALLACE, R.D. Treatment wetlands. 2nd ed. Boca Raton: CRC Press, 2009. 1016p.

[6] APHA - American Public Health Association. Standard Methods for the Examination of Water and Wastewater. 21th. Washington: APHA-AWWA-WEF, 2006

[7] METCALF e EDDY. Wastewater Engineering: Treatment, Disposal and Reuse. 3 ed. New York, USA: Metcalf \& Eddy, Inc. 1991. 1334p.

[8] TSUTIYA, M. T., SOBRINHO, P. A. Coleta e Transporte de Esgoto Sanitário. $3^{\mathrm{a}}$ edição. Rio de Janeiro: ABES - Associação Brasileira de Engenharia Sanitária e Ambiental. 2011. 548 p.

[9] ASSOCIAÇÃO BRASILEIRA DE NORMAS TÉCNICAS. NBR 9649: Projeto de redes coletoras de esgoto sanitário. Rio de Janeiro: ABNT, 1986. 13 p

[10] MATOS, M. P. Colmatação em sistemas alagados construídos de escoamento horizontal subsuperficial: principais fatores e métodos de identificação em unidades plantadas e não plantadas. 2015. 301p Tese (Programa de Pós-graduação em Saneamento, Meio Ambiente e Recursos Hídricos- Doutorado). Universidade Federal de Minas Gerais. Belo Horizonte.

[11] BAPTESTINI, G. C. F.et al. Efeito da inversão no sentido do escoamento em sistemas alagados construídos na produtividade do Cynodon spp. e Alternanthera 
philoxeroides. In: $2^{\circ}$ Simpósio Brasileiro Sobre Wetlands Construídos, 2015, Curitiba. Anais eletrônicos.Curitiba, 2015.

[12] ASSOCIAÇÃO BRASILEIRA DE NORMAS TÉCNICAS. NBR 9649:Projeto de redes coletoras de esgoto sanitário. Rio de Janeiro: ABNT, 1986. $13 \mathrm{p}$.

[13] SUNTTI, C.; TREIN, C. M.; RIBEIRO, E. F.; SCARATTI, D.; SEZERINO. P. H.; Sistemas híbridos de filtros plantados - fluxo vertical seguido de fluxo horizontal para pós-tratamento de esgoto doméstico de um centro de treinamento. In: $1^{\circ}$ Simpósio Brasileiro Sobre Aplicação de Wetlands Construídos no Tratamento de Águas Residuárias, 2013, Florianópolis. Anais eletrônicos. Florianópolis, 2013.

[14] IWA Special Group on Use of Macrophytes in Water Pollution Control (2000). Constructed wetlands for pollutant control:Processes, Performance, design and operation. Scientific and Technology Report, No. 8. IWA Publishing, London, England.

[15] MIRANDA, S. T. Avaliação de aspectos da colmatação e descolmatação de sistemas alagados construídos de escoamento horizontal subsuperficial. 72p. 2014. Dissertação (Programa de Pós Graduação em Recursos Hídricos e AmbientaisMestrado, Universidade Federal de Viçosa, Minas Gerais.

[16] PAOLI, A. C.; VON SPERLING, M. Evaluation of clogging in planted and unplanted horizontal subsurface flow constructed wetlands: solids accumulation and hydraulic conductivity reduction. Water Science and Technology. Vol.67, n.6, p. 1345-1352, 2013.

[17] TREIN, C. M.; PELISSARI, C.; HOFFMANN, H.; PlATZER, C. J.; SEZERINO, P. H. Tratamento descentralizado de esgotos de empreendimentos comercial e residencial empregando a ecotecnologia dos wetlands construídos. Ambiente Construído, Porto Alegre, v. 15, n. 4, p. 351-367, 2015. 